\title{
Dispersion theoretical analysis of the nucleon form factors
}

\author{
Hans-Werner Hammer ${ }^{1,2, \star}$ and Ulf-G. Meißner ${ }^{3,4, \star \star}$ \\ ${ }^{1}$ Institut für Kernphysik, Technische Universität Darmstadt, 64289 Darmstadt, Germany \\ ${ }^{2}$ ExtreMe Matter Institute EMMI, GSI Helmholtzzentrum für Schwerionenforschung GmbH, 64291 Darmstadt, Germany \\ ${ }^{3}$ Universität Bonn, Helmholtz Institut für Strahlen- und Kernphysik, Bethe Center for Theoretical Physics, D-53115 Bonn, Germany \\ ${ }^{4}$ Forschungszentrum Jülich, Institute für Kernphysik (IKP-3), Institute for Advanced Simulation (IAS-4) and Jülich Center for \\ Hadron Physics, D-52145 Jülich, Germany
}

\begin{abstract}
We review the achievements of the project C.2, that deals with a dispersion theoretical analysis of the nucleon form factors. We have analyzed the world data for the space-like and time-like form factors to extract nucleon radii and vector meson coupling constants. Our proton charge radius $r_{E}^{p} \simeq 0.85 \mathrm{fm}$ has gained prominence in the so-called proton radius puzzle, see Ref. [1]. We also have analyzed the strong energydependence of the $e^{+} e^{-} \rightarrow \bar{p} p$ amplitude at the nucleon-antinucleon threshold, two-photon exchange effects and isospin violation in the isovector nucleon form factors.
\end{abstract}

\section{Introduction and motivation}

The project C.2 has been funded just for the first funding period of the SFB/TR 16 . It grew out of an "Einzelverfahren" of the DFG and was then included in the SFB/TR 16. Despite many interesting results, foreseeing e.g. the much debated proton radius puzzle, it was terminated after one funding period. The PIs were HansWerner Hammer and Ulf Meißner, who have collaborated on the dispersive analysis of the nucleon form factors since 1996.

The project C.2 dealt with the dispersion-theoretical analysis of the nucleon form factors. These encode important information about perturbative and non-perturbative aspects of QCD manifest in the nucleon structure. They are not only interesting by themselves, but a precise knowledge is mandatory for many other experiments such as the measurement of the nucleon's strange form factors and fundamental tests of Quantum Electrodynamics. Dispersion relations provide a powerful tool to analyze the form factors in a model-independent way and include constraints from multi-meson continua and chiral perturbation theory. In this project, we have made considerable progress towards our goal of understanding the nucleon structure as parameterized by the form factors. We have reanalyzed the contribution of the $2 \pi$ continuum and included the contributions from the $K \bar{K}$ and $\rho \pi$ continua to constrain the spectral functions. For the first time, we have given an estimate for the theoretical errors in the extracted form factors. The form factor data in the spaceand time-like regions are well described within our error bands. The strong rise of the time-like form factors could

\footnotetext{
${ }^{\star}$ e-mail: hammer@theorie.ikp.physik.tu-darmstadt.de

${ }^{\star}$ e-mail: meissner@hiskp.uni-bonn.de
}

be explained by well-known properties of the nucleonantinucleon $(N \bar{N})$ interaction. Finally, we have quantified the effects of isospin violation in the nucleon form factors, which is an important ingredient in the extraction of strange nucleon form factors from the measurements of parity violating asymmetries in electron-proton scattering.

Our contribution is organized as follows: Section 2 summarizes the results on the isovector spectral functions of the nucleon formfactors. Based on this, a fullfledged dispersive analysis including meson continua was performed, as summarized in Sec. 3. The strong energy dependence of the $e^{+} e^{-} \rightarrow p \bar{p}$ amplitude near threshold is analyzed in Sec. 4, followed by a model-independent calculation of the so important two-photon corrections, see Sec. 5. Finally, isospin violation in the vector form factors of the nucleon is discussed in Sec. 6. For convenience, we collect some basic definitions in the Appendix.

Note the important disclaimer: This is the review of the project C.2 but not a general review of the various fields to which this research contributed. Therefore, only the papers that resulted from this research are quoted, with the exception of a few external papers and of course, in case data were involved, with proper reference to the experimental papers. The links to the other projects are mentioned in passing, but no effort is made to discuss these in any detail.

\section{Novel evaluation of the two-pion contribution to the nucleon isovector form factors}

The spectral functions of the nucleon form factors are constrained by input from meson continua. The most important constraint on the isovector spectral function comes 


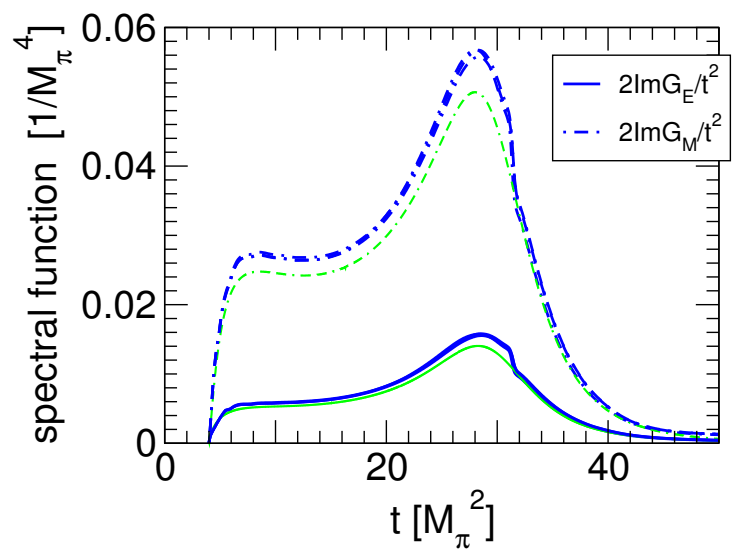

Figure 1. The $2 \pi$ spectral function using the new high statistics data for the pion form factor [3-5]. The spectral functions weighted by $1 / t^{2}$ are shown for $G_{E}$ (solid line) and $G_{M}$ (dashdotted line). The previous results by Höhler et al. [6] (without $\rho-\omega$ mixing) are shown for comparison by the gray/green lines.

from the $2 \pi$ continuum. Approximating this continuum by a simple $\rho$ pole leads to a severe underestimation of the isovector radii of the nucleon. This contribution was evaluated more than 40 years ago be Höhler and Pietarinen. We have performed a new calculation of this contribution drawing upon the new high statistics measurements of the pion form factor by the CMD-2, KLOE, and SND collaborations, see Fig. 1. The general structure of the spectral functions remains unchanged, but the magnitude increases by about $10 \%$. Using the updated spectral functions, we have calculated the contribution of the $2 \pi$ continuum to the nucleon isovector form factors and radii. Moreover, we have given a convenient parametrization of the result for use in future form factor analyses [2]. For the isoscalar spectral functions, we rely on the determinations of the $K \bar{K}$ and $\rho \pi$ continua in the literature.

\section{Dispersion analysis of the nucleon form factors including meson continua}

Dispersion theory simultaneously describes all four nucleon form factors over the whole range of momentum transfers in both the space-like and time-like regions. It allows for the inclusion of constraints from other physical processes, unitarity, and chiral perturbation theory and therefore is an ideal tool to analyze the form factor data. Using the $2 \pi$ continuum discussed in the previous section as well the $K \bar{K}[9,10]$ and $\rho \pi$ continua [11] as input, we have analyzed the world data for the electromagnetic form factors of the nucleon in the space- and time-like regions $[7,8]$. The central objects of the dispersive analysis of the nucleon form factors are the spectral functions, i.e. the imaginary parts of the form factors given by

$$
F(t)=\frac{1}{\pi} \int_{t_{0}}^{\infty} \frac{\operatorname{Im} F\left(t^{\prime}\right)}{t^{\prime}-t-i \epsilon} d t^{\prime},
$$

where $t_{0}$ is the threshold of the lowest cut of $F(t)$ and the $i \epsilon$ defines the integral for values of $t$ on the cut. In all our fits
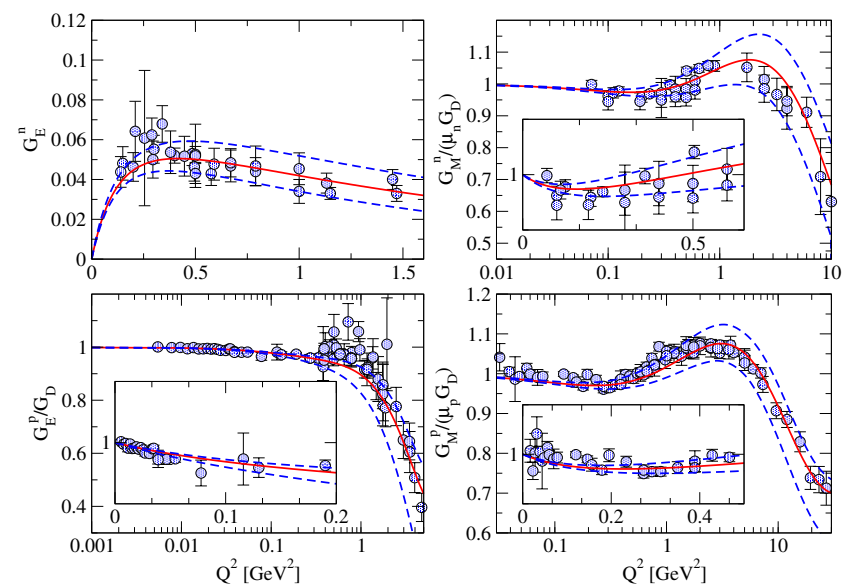

Figure 2. The nucleon electromagnetic form factors for spacelike momentum transfer in the SC approach. The solid line gives our best fit while the dashed lines indicate the $1 \sigma$ error band.

the spectral function includes the $2 \pi, K \bar{K}$, and $\rho \pi$ continua from unitarity and the $\omega$ pole. Note that we also include a pole at the $\phi$ mass to account for explicit $\phi$ strength not included in the $K \bar{K}$ and $\rho \pi$ continua. In addition to that there are a number of effective poles at higher momentum transfers in the isoscalar $\left(s_{1}, s_{2}, \ldots\right)$ and isovector channels $\left(v_{1}, v_{2}, \ldots\right)$. Note that for the dispersive analysis, the isospin basis is most natural rather than the particle basis. The spectral function has the general structure

$$
\begin{aligned}
\operatorname{Im} F_{i}^{s}(t)= & \operatorname{Im} F_{i}^{(s, K \bar{K})}(t)+\operatorname{Im} F_{i}^{(s, \rho \pi)}(t) \\
& +\sum_{V=\omega, \phi, s_{1}, \ldots} \pi a_{i}^{V} \delta\left(M_{V}^{2}-t\right), \quad i=1,2, \\
\operatorname{Im} F_{i}^{v}(t)= & \operatorname{Im} F_{i}^{(v, 2 \pi)}(t) \\
& +\sum_{V=v_{1}, \ldots} \pi a_{i}^{V} \delta\left(M_{V}^{2}-t\right), \quad i=1,2 .
\end{aligned}
$$

The vector meson residua $a_{i}^{V}$ (for the $\omega, \phi, s_{i}$ and $v_{i}$ ) are fit parameters. Constraints are derived from the normalizations of the four form factors at zero momentum transfer as well as from the perturbative QCD behavior of the form factors. We have used two different methods to include the asymptotic constraints from perturbative QCD: (a) applying superconvergence relations only (SC) and (b) adding an explicit pQCD term to the spectral function. The results for the form factors were similar in both approaches. In general, we have 14 - 16 fit parameters, for details see Ref. [8]. Moreover, we have provided a $1 \sigma$ error band for our analysis and generally found good agreement with the world data within the errors. This method to generate theoretical errors was later used in other projects, like B.3 and B.6. As an example, we show our best fit in the SC approach in Figs. 2 and 3. In particular, no evidence for a bump-dip-structure in $G_{E}^{n}$ was found [21]. We have also extracted the nucleon radii, see Tab. 1 , and the $\omega N N$ coupling constants. For the radii, we generally found good agreement with other determinations with the exception of the electric charge radius of the proton which comes out smaller. While the Lamb shift in electronic hydrogen 
Table 1. Nucleon radii extracted from our fits in the SC (2nd column) and explicit pQCD approaches (3rd column). The first number gives the value for our best fit, while the numbers in parentheses indicate the range from the $1 \sigma$ band. For comparison, we give the results of Ref. [12] (4th column) and other recent determinations from low-momentum transfer data [13-18] (5th column).

\begin{tabular}{|c|c|c|c|c|}
\hline & SC approach & explicit pQCD app. & Ref. [12] & recent determ. \\
\hline \hline$r_{E}^{p}[\mathrm{fm}]$ & $0.844(0.840 \ldots 0.852)$ & $0.830(0.822 \ldots 0.835)$ & 0.848 & $0.886(15)[13-15]$ \\
$r_{M}^{p}[\mathrm{fm}]$ & $0.854(0.849 \ldots 0.859)$ & $0.850(0.843 \ldots 0.852)$ & 0.857 & $0.855(35)[14,16]$ \\
$\left(r_{E}^{n}\right)^{2}\left[\mathrm{fm}^{2}\right]$ & $-0.117(-0.11 \ldots-0.128)$ & $-0.119(-0.108 \ldots-0.13)$ & -0.12 & $-0.115(4)[17]$ \\
$r_{M}^{n}[\mathrm{fm}]$ & $0.862(0.854 \ldots 0.871)$ & $0.863(0.859 \ldots 0.871)$ & 0.879 & $0.873(11)[18]$ \\
\hline
\end{tabular}

gives $r_{E}^{p} \simeq 0.88 \mathrm{fm}$, we find a "small" radius in the range from $0.84 \ldots 0.85 \mathrm{fm}$. We are not able to obtain good fits when we force the proton charge radius to the large value. This has preceded the now famous "proton radius puzzle" by many years and was unfortunately not appreciated by the reviewers of this project. The $\omega N N$ vector coupling constant is determined relatively well by the fits, but for the tensor coupling constant even the sign can not be determined. Some challenges remain in the time-like region.
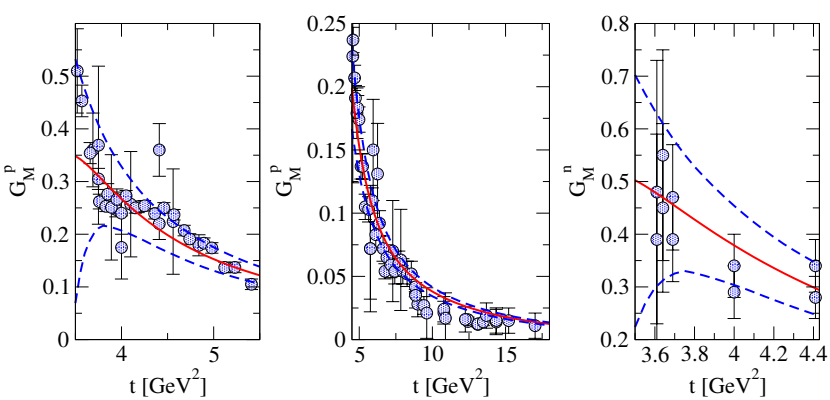

Figure 3. Left panel: The nucleon electromagnetic form factors for time-like momentum transfer in the SC approach (right panel: $G_{M}^{n}$, left panels: $G_{M}^{p}$ ). The proton data participate in the fit while the neutron data are a genuine prediction. The solid line gives our best fit while the dashed lines indicate the $1 \sigma$ error band.

\section{On the strong energy dependence of the $e^{+} e^{-} \rightarrow p \bar{p}$ amplitude near threshold}

It is often claimed that the strong energy dependence of the nucleon time-like form factors, cf. Fig. 3, requires the presence of a new resonance in the $N \bar{N}$-threshold region. In order to resolve this puzzle, we have studied the energy dependence of the $e^{+} e^{-} \rightarrow p \bar{p}$ cross section close to the two-nucleon threshold, recently reported by the BaBar collaboration. Our analysis also included the $\bar{p} p \rightarrow e^{+} e^{-}$data collected by PS170 collaboration and the $e^{+} e^{-} \rightarrow N \bar{N}$ data from the FENICE collaboration. We have shown that the near-threshold enhancement in the $e^{+} e^{-} \rightarrow p \bar{p}$ cross section can be explained by the finalstate interaction between proton and antiproton in the ${ }^{3} S_{1}$ partial wave [22], utilizing the Jülich nucleon-antinucleon model [23, 24], see Fig. 4. As a consequence, the strong dependence of the proton electromagnetic form factors on the momentum transfer close to the two-nucleon threshold is then likewise driven by this well-known final-state interaction effect. This result is in line with our previous studies $[25,26]$ of the near-threshold enhancement of the $p \bar{p}$ invariant mass spectrum seen in the $J / \Psi \rightarrow \gamma p \bar{p}$ decay by the BES collaboration and in the $B^{+} \rightarrow p \bar{p} K^{+}$decay by the BaBar collaboration.

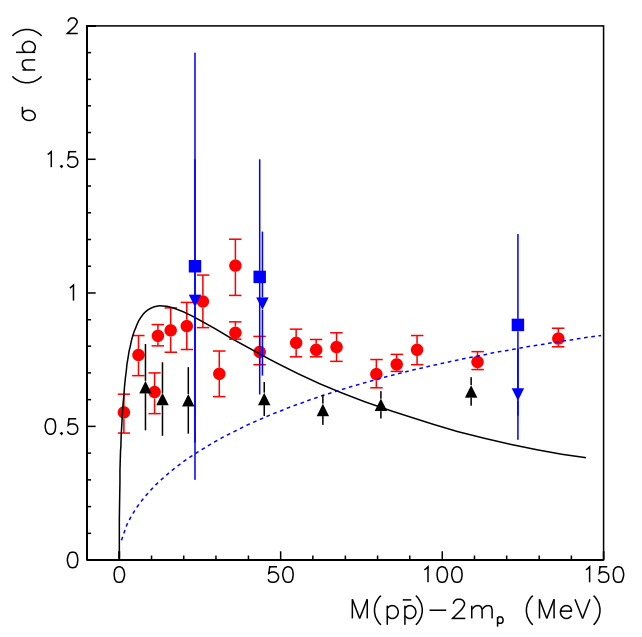

Figure 4. Cross section of the $e^{+} e^{-} \rightarrow p \bar{p}$ and $e^{+} e^{-} \rightarrow n \bar{n}$ reactions as a function of the excess energy. The data are from the FENICE (inverse triangles and squares) [27, 28] and BaBar (circles) collaborations [29]. Triangles represent results obtained by applying detailed balance to the $\bar{p} p \rightarrow e^{+} e^{-}$cross section measured by the PS170 collaboration [30]. The dashed line indicates the energy dependence of the two-body phase space. The solid line is the scattering amplitude squared predicted by the Jülich $N \bar{N}$ model A(OBE) for the ${ }^{3} S_{1}$ partial wave, multiplied by appropriate phase-space factors.

\section{A model-independent estimate of two-photon effects in unpolarized elastic electron-proton scattering}

In order to investigate the importance of two-photon effects we have directly analyzed the experimental cross sections for the proton. When the discrepancy between the Rosenbluth and the polarization transfer measurement results was first observed, it was noted that the values of $G_{E}^{p}$ extracted using the Rosenbluth technique are not consistent with each other. It was often assumed that the difference between the results of the two techniques can be explained by systematic uncertainties in the Rosenbluth 

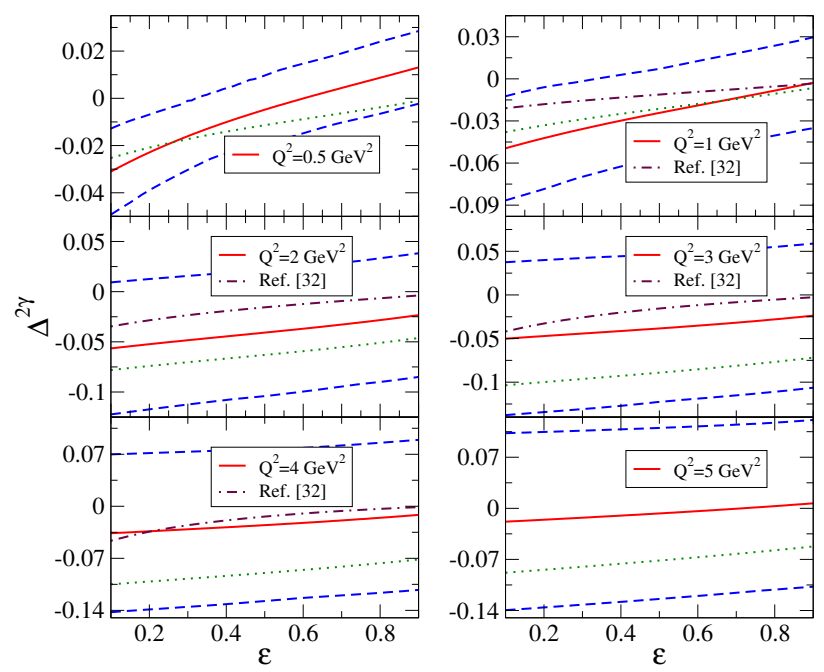

Figure 5. The extracted two-photon correction $\Delta^{2 \gamma}$ for $Q^{2}=0.5$, $1.0,2.0,3.0,4.0$, and $5.0 \mathrm{GeV}^{2}$. Our correction $\Delta^{2 \gamma}$ corresponds to the sum of the extracted hard two-photon correction $\delta^{2 \gamma}$ and the Coulomb correction $\delta^{C}$. The solid lines show our results for $\Delta^{2 \gamma}$ within the SC approach, while the dashed lines gives the theoretical $1 \sigma$ uncertainty bands. The dotted lines give the central value for $\Delta^{2 \gamma}$ within the $\mathrm{pQCD}$ approach. For comparison, the direct full calculation by Blunden et al. [32] is shown by the dashdotted lines.

extractions. A global reanalysis of the Rosenbluth data showed that only the data from individual experiments are consistent but the discrepancy persisted. Applying the standard radiative corrections consistently, we have performed a global analysis of the Rosenbluth cross section data for the proton and form factor data for the neutron. Comparing the result for the proton (electron-proton scattering) to the polarization data for the proton, we have obtained an estimate of the two-photon corrections defined by

$$
\left(\frac{d \sigma}{d \Omega}\right)_{\operatorname{Ros}}=\left(\frac{d \sigma}{d \Omega}\right)\left(1+\delta^{2 \gamma}\right),
$$

in Ref. [31], with $(d \sigma / d \Omega)_{\text {Ros }}$ the standard Rosenbluth cross section in terms of the electric and magnetic nucleon form factors. The full two-photon correction $\Delta^{2 \gamma}$ is obtained by adding the Coulomb correction $\delta^{C}$. The results from our work are in good agreement with the direct calculations of the two-photon exchange corrections by Blunden et al. [32] but the error bars leave room for improvement, see Fig. 5. Our results also show that the discrepancy between the Rosenbluth and the polarisation transfer measurement results becomes significant only above $Q^{2} \approx 4 \mathrm{GeV}^{2}$.

\section{Isospin violation in the vector form factors of the nucleon}

A quantitative understanding of isospin violation is an increasingly important ingredient for the extraction of the nucleon's strange vector form factors from experimental data. We have calculated the isospin violating electric and magnetic form factors in chiral perturbation theory to leading and next-to-leading order, respectively [33]. We have extracted the low-energy constants from resonance saturation using the $\omega N N$ coupling constants from our dispersion analysis [8]. The uncertainties in the isospin violating form factors are largely dominated by limitations in the current knowledge of some vector meson couplings. The resulting bounds on isospin violation are sufficiently precise to be of value to on-going experimental studies of the strange form factors. As an example, we show the isospin breaking form factor $G_{E}^{u, d}(t)$ in Fig. 6.

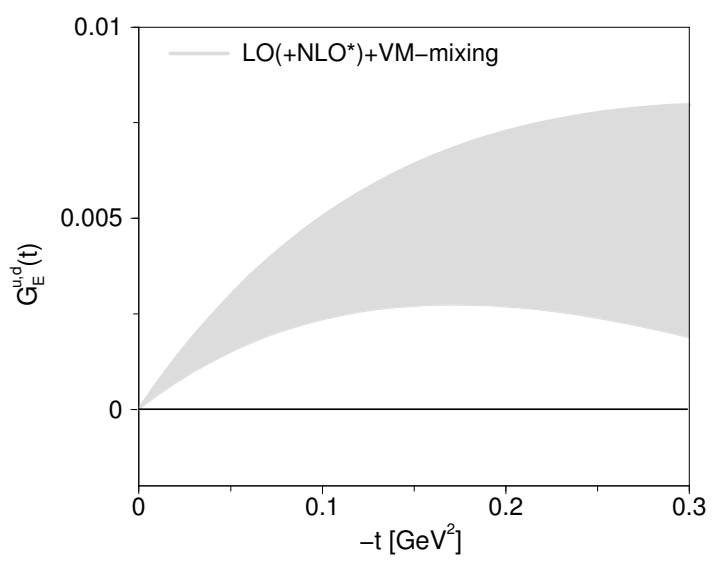

Figure 6. The isospin breaking form factor $G_{E}^{u, d}(t)$. The band combines uncertainties in the vector meson mixing amplitude as well as from higher-order chiral corrections.

We would like to thank our collaborators Maxim Belushkin, Johan Haidenbauer, Siegfried Krewald, Anthony Thomas, Randy Lewis and the late Alexander Sibirtsev. The work reported here would not have been possible without the support from the Deutsche Forschungsgemeinschaft within the SFB/TR16.

\section{A Basic definitions}

The electromagnetic (em) structure of the nucleon is determined by the matrix element of the vector current operator $j_{\mu}^{\mathrm{em}}$ between nucleon states. Using Lorentz and gauge invariance, this matrix element can be expressed in terms of two form factors,

$$
\left\langle p^{\prime}\left|j_{\mu}^{\mathrm{em}}\right| p\right\rangle=\bar{u}\left(p^{\prime}\right)\left[F_{1}(t) \gamma_{\mu}+i \frac{F_{2}(t)}{2 M} \sigma_{\mu \nu} q^{\nu}\right] u(p),
$$

where $M$ is the nucleon mass and $t=\left(p^{\prime}-p\right)^{2}$ the fourmomentum transfer squared. For data in the space-like region, it is often convenient to use the variable $Q^{2}=-t>$ 0 . The functions $F_{1}(t)$ and $F_{2}(t)$ are the Dirac and Pauli form factors, respectively. They are normalized at $t=0$ as

$$
F_{1}^{p}(0)=1, F_{1}^{n}(0)=0, F_{2}^{p}(0)=\kappa_{p}, F_{2}^{n}(0)=\kappa_{n},
$$

with $\kappa_{p}=1.793$ and $\kappa_{n}=-1.913$ the anomalous magnetic moment of the proton and the neutron, respectively, in units of nuclear magnetons.

It is convenient to work in the isospin basis and to decompose the form factors into isoscalar and isovector 
parts,

$$
F_{i}^{s}=\frac{1}{2}\left(F_{i}^{p}+F_{i}^{n}\right), \quad F_{i}^{v}=\frac{1}{2}\left(F_{i}^{p}-F_{i}^{n}\right),
$$

where $i=1,2$. The experimental data are usually given for the Sachs form factors

$$
\begin{aligned}
G_{E}(t) & =F_{1}(t)-\tau F_{2}(t), \\
G_{M}(t) & =F_{1}(t)+F_{2}(t),
\end{aligned}
$$

where $\tau=-t /\left(4 M^{2}\right)$. In the Breit frame, $G_{E}$ and $G_{M}$ may be interpreted as the Fourier transforms of the charge and magnetization distributions, respectively.

The nucleon radii $\sqrt{\left\langle r^{2}\right\rangle}$ can be defined from the low- $t$ expansion of the form factors,

$$
F(t)=F(0)\left[1+t\left\langle r^{2}\right\rangle / 6+\ldots\right],
$$

where $F(t)$ is a generic form factor. In the case of the electric and Dirac form factors of the neutron, $G_{E}^{n}$ and $F_{1}^{n}$, the expansion starts with the term linear in $t$ and the normalization factor $F(0)$ is dropped.

\section{References}

[1] R. Pohl et al., Nature 466 (2010) 213.

[2] M. A. Belushkin, H.-W. Hammer and U.-G. Meißner, Phys. Lett. B 633 (2006) 507 [arXiv:hep-ph/0510382].

[3] R. R. Akhmetshin et al. [CMD-2 Collaboration], arXiv:hep-ex/9904027, Phys. Lett. B 527 (2002) 161 [arXiv:hep-ex/0112031], Phys. Lett. B 578 (2004) 285 [arXiv:hep-ex/0308008].

[4] A. Aloisio et al. [KLOE Collaboration], Phys. Lett. B 606 (2005) 12 [arXiv:hep-ex/0407048].

[5] M. N. Achasov et al., J. Exp. Theor. Phys. 101 (2005) 1053 [Zh. Eksp. Teor. Fiz. 101 (2005) 1201] [hepex/0506076].

[6] G. Höhler, "Pion-Nucleon Scattering", LandoltBörnstein Vol. I/9b, ed. H. Schopper, Springer, Berlin, 1983.

[7] H.-W. Hammer, Eur. Phys. J. A 28, s01 (2006) 49 [arXiv:hep-ph/0602121].

[8] M. A. Belushkin, H.-W. Hammer and U.-G. Meißner, Phys. Rev. C 75 (2007) 035202 [arXiv:hepph/0608337].

[9] H.-W. Hammer and M.J. Ramsey-Musolf, Phys. Rev. C 60, 045205 (1999) [Erratum-ibid. C 62, 049903 (2000)] [arXiv:hep-ph/9812261].

[10] H.-W. Hammer and M.J. Ramsey-Musolf, Phys. Rev. C 60, 045204 (1999) [Erratum-ibid. C 62, 049902 (2000)] [arXiv:hep-ph/9903367].
[11] U.-G. Meißner, V. Mull, J. Speth and J. W. van Orden, Phys. Lett. B 408, 381 (1997) [arXiv:hep$\mathrm{ph} / 9701296]$.

[12] H.-W. Hammer and U.-G. Meißner, Eur. Phys. J. A 20, 469 (2004) [arXiv:hep-ph/0312081].

[13] R. Rosenfelder, Phys. Lett. B 479, 381 (2000) [arXiv:nucl-th/9912031].

[14] I. Sick, Phys. Lett. B 576, 62 (2003) [arXiv:nuclex/0310008].

[15] K. Melnikov and T. van Ritbergen, Phys. Rev. Lett. 84, 1673 (2000) [arXiv:hep-ph/9911277].

[16] I. Sick, private communication.

[17] S. Kopecky, M. Krenn, P. Riehs, S. Steiner, J. A. Harvey, N. W. Hill, and M. Pernicka, Phys. Rev. C 56, 2229 (1997).

[18] G. Kubon et al., Phys. Lett. B 524, 26 (2002) [arXiv:nucl-ex/0107016].

[19] P.G. Blunden and I. Sick, Phys. Rev. C 72, 057601 (2005) [arXiv:nucl-th/0508037].

[20] J. Arrington, private communication.

[21] U.-G. Meißner, AIP Conf. Proc. 904 (2007) 142 [nucl-th/0701094].

[22] J. Haidenbauer, H.-W. Hammer, U.-G. Meißner and A. Sibirtsev, Phys. Lett. B 643 (2006) 29 [arXiv:hep$\mathrm{ph} / 0606064]$.

[23] T. Hippchen, J. Haidenbauer, K. Holinde, V. Mull, Phys. Rev. C 44, 1323 (1991); V. Mull, J. Haidenbauer, T. Hippchen, K. Holinde, Phys. Rev. C 44, 1337 (1991).

[24] V. Mull and K. Holinde, Phys. Rev. C 51 (1995) 2360 [nucl-th/9411014].

[25] A. Sibirtsev, J. Haidenbauer, S. Krewald, U.G. Meißner and A. W. Thomas, Phys. Rev. D 71 (2005) 054010 [arXiv:hep-ph/0411386].

[26] J. Haidenbauer, U.-G. Meißner and A. Sibirtsev, Phys. Rev. D 74 (2006) 017501 [arXiv:hep$\mathrm{ph} / 0605127]$.

[27] A. Antonelli et al., Phys. Lett. B 334, 431 (1994).

[28] A. Antonelli et al., Nucl. Phys. B 517, 3 (1998).

[29] B. Aubert et al., Phys. Rev. D 73 (2006) 012005 [hepex/0512023].

[30] G. Bardin et al., Nucl. Phys B 411 (1994) 3.

[31] M. A. Belushkin, H.-W. Hammer and U.-G. Meißner, Phys. Lett. B 658 (2008) 138 [arXiv:0705.3385 [hep$\mathrm{ph}]]$.

[32] P. G. Blunden, W. Melnitchouk and J. A. Tjon, Phys. Rev. C 72 (2005) 034612 [arXiv:nucl-th/0506039].

[33] B. Kubis and R. Lewis, Phys. Rev. C 74 (2006) 015204 [arXiv:nucl-th/0605006]. 